\title{
Optimized Features Extraction of IRIS Recognition by Using MADLA to Ensure Secure Authentication
}

\author{
S. Pravinthraja ${ }^{1}$, K. Umamaheswari ${ }^{2}$ \\ ${ }^{1}$ Sri Ramakrishna Institute of Technology, Coimbatore, India \\ ${ }^{2}$ PSG College of Technology, Coimbatore, India \\ Email: pravinthraja@gmail.com
}

Received 1 March 2016; accepted 25 March 2016; published 27 June 2016

Copyright (C) 2016 by authors and Scientific Research Publishing Inc.

This work is licensed under the Creative Commons Attribution International License (CC BY). http://creativecommons.org/licenses/by/4.0/

(c) (i) Open Access

\begin{abstract}
Nowadays, Iris recognition is a method of biometric verification of the person authentication process based on the human iris unique pattern, which is applied to control system for high level security. It is a popular system for recognizing humans and essential to understand it. The objective of this method is to assign a unique subject for each iris image for authentication of the person and provide an effective feature representation of the iris recognition with the image analysis. This paper proposed a new optimization and recognition process of iris features selection by using proposed Modified ADMM and Deep Learning Algorithm (MADLA). For improving the performance of the security with feature extraction, the proposed algorithm is designed and used to extract the strong features identification of iris of the person with less time, better accuracy, improving performance in access control and in security level. The evaluations of iris data are demonstrated the improvement of the recognition accuracy. In this proposed methodology, the recognition of the iris features has been improved and it incorporates into the iris recognition systems.
\end{abstract}

\section{Keywords}

GLCM, Deep Learning, Strong Features Extraction, MADMM, Iris Recognition

\section{Introduction}

For years, recognition of bodily features has been used for the purpose of identification and security such as the face, fingerprint, and iris. As a result of progress in the field of iris recognition, it recognizes the structure to regulate the eye sizes and eye surrounding of the pupil. Recently, several studies are made related to the use of 
recognition of iris model to support biometric experiments. It illustrates the iris biometrics matching, where different data are employed for training and testing the enrollment, and often leads to reduced performance. It stimulates the data from database that are acquired under getting hold of heavily controlled conditions.

The Unique Identification Authority of India is enrolling the people per day about one million, at 40,000 stations, and plans to enroll the people within 3 years about 1.2 billion of population. IRIS recognition has materialized as one of the most auspicious technologies to provide reliable identification of human and for noncontact biometric authentication [1]. Also, it became as research topic driven in wide applications like national ID card, border control, banking, etc. Amazing accuracy of iris recognition has been reported by the existing state-ofthe-art iris recognition algorithms from controlled environment.

In human eye, iris is a ring shaped region with biometric patterns. It is stable and provides a reliable approach for individual authentication. Based on automatic preprocessing, features matching and analysis, a unique identity label is assigning to each iris image features for extracting and identification of the person [2]. The state-of-the-art of the iris recognition methods has a unique image differentiation by its feature extraction strategies. In preprocessing, it eliminated the unwanted parts of the image and controlled the pupil function with adjusting the size of the image. The diameter of iris is $12 \mathrm{~mm}$ and pupil size of the diameter will be varying from $10 \%$ to $80 \%$.

In iris recognition applications, iris images are defined with the same class of different subjects [3]. So that the iris images dissimilarity of the subjects is need to be identified for authentication. However, in iris biometrics applications the similarities between various subjects are identified which categorize iris images into several explicit classes. Moreover, the iris images in the central database into multiple categories may help for speed up the iris identification in large-scale. In existing recognition systems, the iris images within the short distance and under constrained environment are obtained using NIR [4] [5]. It achieves the matching accuracy of the image and preserves it clearly in high quality.

For long period of time, the patterns of each person are believed to be unique and remain stable and make them as a biometric signature. Over the past decade, iris patterns have gone through significant transformations of developing a new one and upgrading an existing. Sometimes, the enrollment is time consuming and expensive by having large number of users in the hundreds of millions. This makes it infeasible in deploying to reenroll users every time. In practice, iris images for enrollment are trained and tested.

The paper is organized as follows. Section 2 reviews the most appropriate approaches for the amalgamation of iris data. Section 3 provides the proposed method description with validates of synthesis and interpreting strategies used along with the observed factors of unpredictability data. Section 4 presents an analysis of experimental results of proposed work with its strategies and parameters. Finally, conclusions and future work are given in Section 5 .

\section{Related Work}

In this section the survey related to the iris recognition system is discussed. A novel optimization framework is proposed for learning iris biometrics transformations with its desired properties and represented concisely using kernel functions [6]. The proposed work is utilized for high adaptation by performing it in a similar way with limiting the samples from various data's set. Furthermore, the classes will be large between various samples and these constraints make sure that alleviated the mismatch problem when matching is performed.

For extracting the features and solving of optimization the Alternating Direction Method of Multiplexer (ADMM) is used [7]. It is easy to handle and solve the issues by breaking them in to smaller pieces. Through this ADMM algorithm, the iris images is divided into several pieces among the whole pixel values and selecting only few regions of images using this optimization algorithm for strong feature extraction.

In order to reduces the complexity and eliminate the insignificant features a new optimization process is designed and implemented. After this GLCM (Gray Level Co-occurrence matrix) algorithm is used to get all the image statistical features so that DBN (Deep Belief network) is train and it is comes under the deep learning family [8]. It is an Advanced Artificial neural network, will learn the input features and respond according to the situation. The extracted features of iris are used in the training process un-supervising and then the trained data is ready to recognize the query of iris image given.

In iris image the function of obtaining accuracy which is based on the method of segmentation. The parameters are iris inner and outer borders [9] [10]. The pupil detection of the iris image is identified with the transfor- 
mation and filtering [11]. The outer border is defined by sobel filtering and the method provides a better performance analysis in dark background iris image by non-concentric iris and pupil.

For obtaining the solution the test samples is need to be perform every time if the optimization problem has a global optimum and convex. This involves in adaptation parameter estimation during the training stage. During testing, the samples are transformed and done matching which leads to significant improvements in accuracy. The components in iris recognition system are strong features extraction, identification, matching and ensure secure recognition for security. In order to overcome the limitation of the existing work, the Modified ADMM and Deep Learning algorithm are proposed for optimizing the extraction of the features for strong features with better performance and accuracy.

\section{Modified ADMM and Deep Learning Algorithm—MADLA}

The proposed work analysis and algorithm are discussed in this section. In this work, the analysis and design of the training phase and testing phase is proposed with the strong features extraction of optimization and preprocessing technique. Modified ADMM and Deep Learning Algorithm are proposed with high level abstraction for ensuring the identification of the person.

\subsection{Training Phase}

In training phase, the process of extracting the iris image is consists from the dataset with large number of iris sample which belongs to different persons. It consist the data set with significant and insignificant information. When processing it required more time consumption for data acquires and also it may lead to confusion during the matching stage. In order to overcome the limitation, a new algorithm (MADMM) is proposed with the optimization and preprocessing.

The proposed MADMM algorithm used to optimize the issues and solved it by dividing large problems in to smaller pieces. It will reduce the image information by eliminating the insignificant features. The extracted features are left to matching stage to find strong features in iris image by using the proposed algorithm (MDLA) of GLCM with DBN algorithm, as shown in Figure 1.

High abstractions features are defined by using the proposed MDLA. For easy identification process, a unique identity label is assigning to each iris image features. This algorithm is capable of learning the features for the purpose of classification. As a solution a trained model is generated with all information of the classifier model.

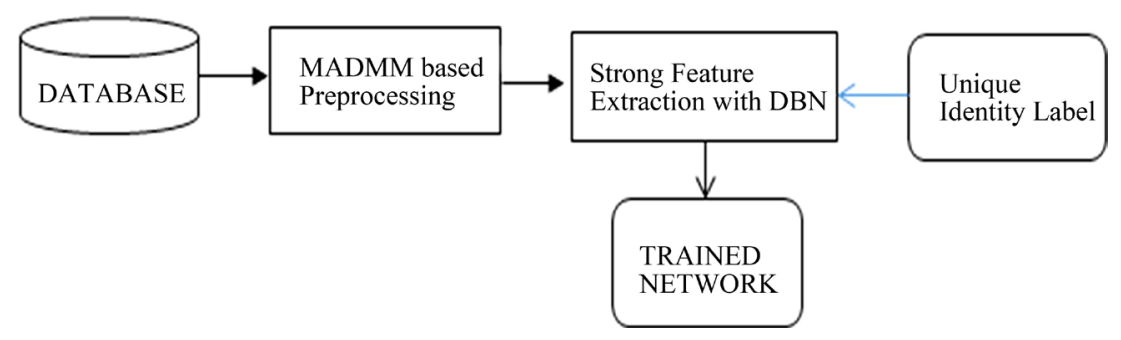

Figure 1. Training phase of MADLA.

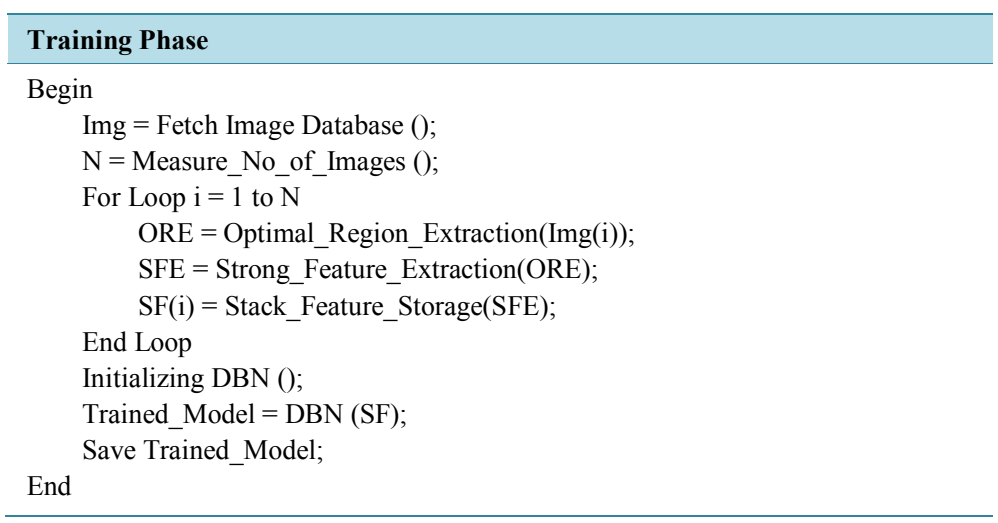




\subsection{Testing Phase}

Testing phase is similar to the training methodology, but instead of database input a query image is given as input. The input query image will undergo the process of proposed MADMM algorithm which is discussed in training phase and the required portion of image data is preserved in it. In tested phase the extracted features is processed with GLCM and analysed the matches with the identity label to find a closest match, as shown in Figure 2.

\section{Experimental Results}

The analyses of experimental results are discussed and compared the MADLA results with the various existing work. The results of extracting strong features take place with the unique identity label. When extracting the query it obtains the iris recognition with identity label which provide secure identification or authentication of a person.

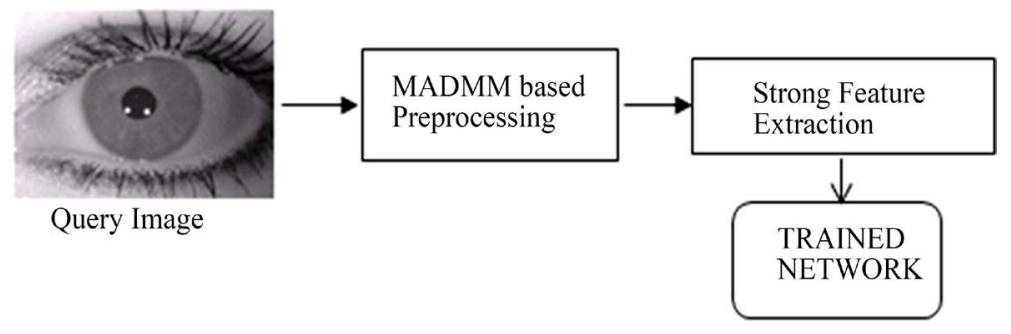

Figure 2. Testing phase of MADLA.

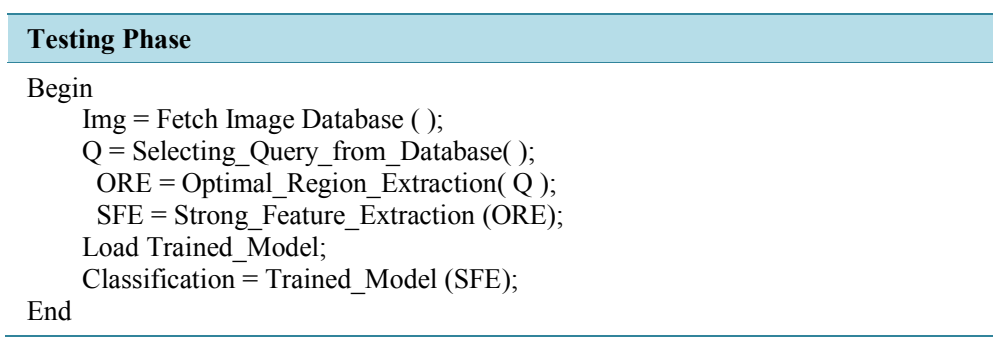

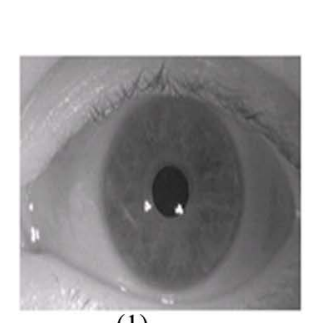

(1)

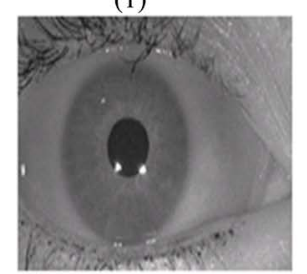

(5)

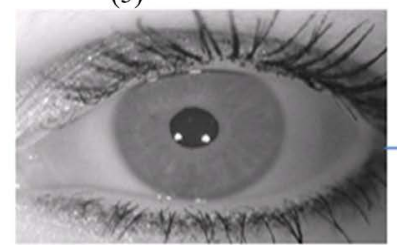

Database Iris Images with its unique identifiers

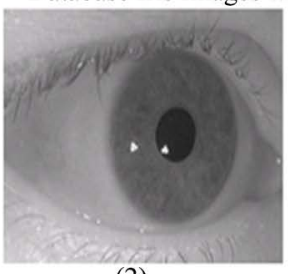

(2)

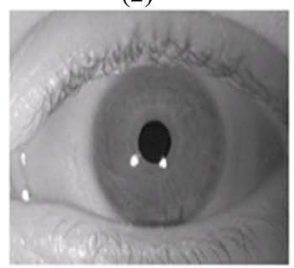

(6)

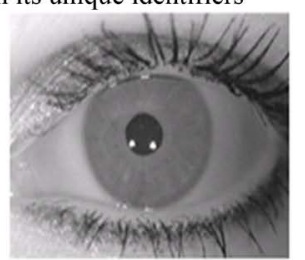

(3)

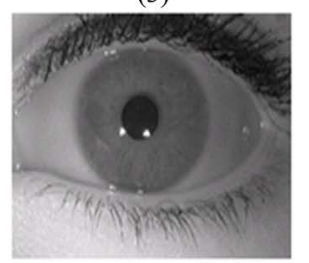

(7)

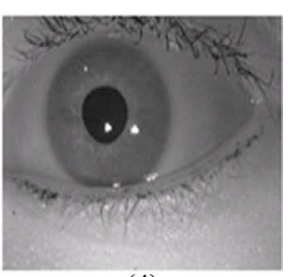

(4)

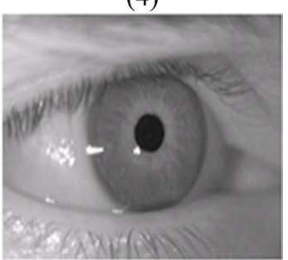

(8)

Figure 3. The results of MADLA recognition system. 
When comparing the work, MADLA provides better accuracy with less time, less error rate, better decidability index and high recognition rate. The data set is taken from IRIS database of Multimedia University (MMU).

As shown in Figure 3, the features extraction results are obtained with identity label. The parameters comparison between the existing methods and proposed method is shown in Figure 4, Figure 5 and Figure 6. Table 1 shows the comparison of the proposed and existing algorithm with the parameter results.

\section{Conclusions}

In proposed iris recognition system, we analyzed the results of the MADLA algorithm to prove the efficiency of the system with less time, better performance and secure authentication, obtaining high level abstraction and better recognition accuracy when compared to existing system. For evaluation of the system, the sample data are enrolled and tested by using the efficient algorithm of MADLA.

The main contributions of this work are to provide a solution for mismatch problem in iris biometrics for improving the matching process feature extraction with better accuracy and less time computation. Future works

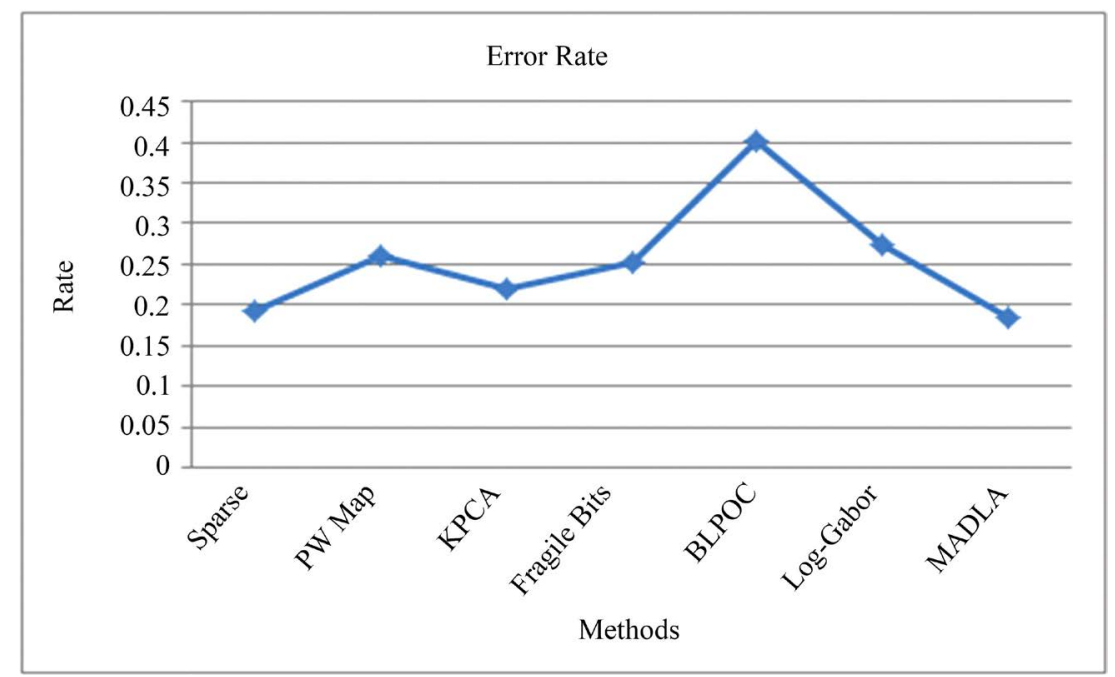

Figure 4. Error rate comparison.

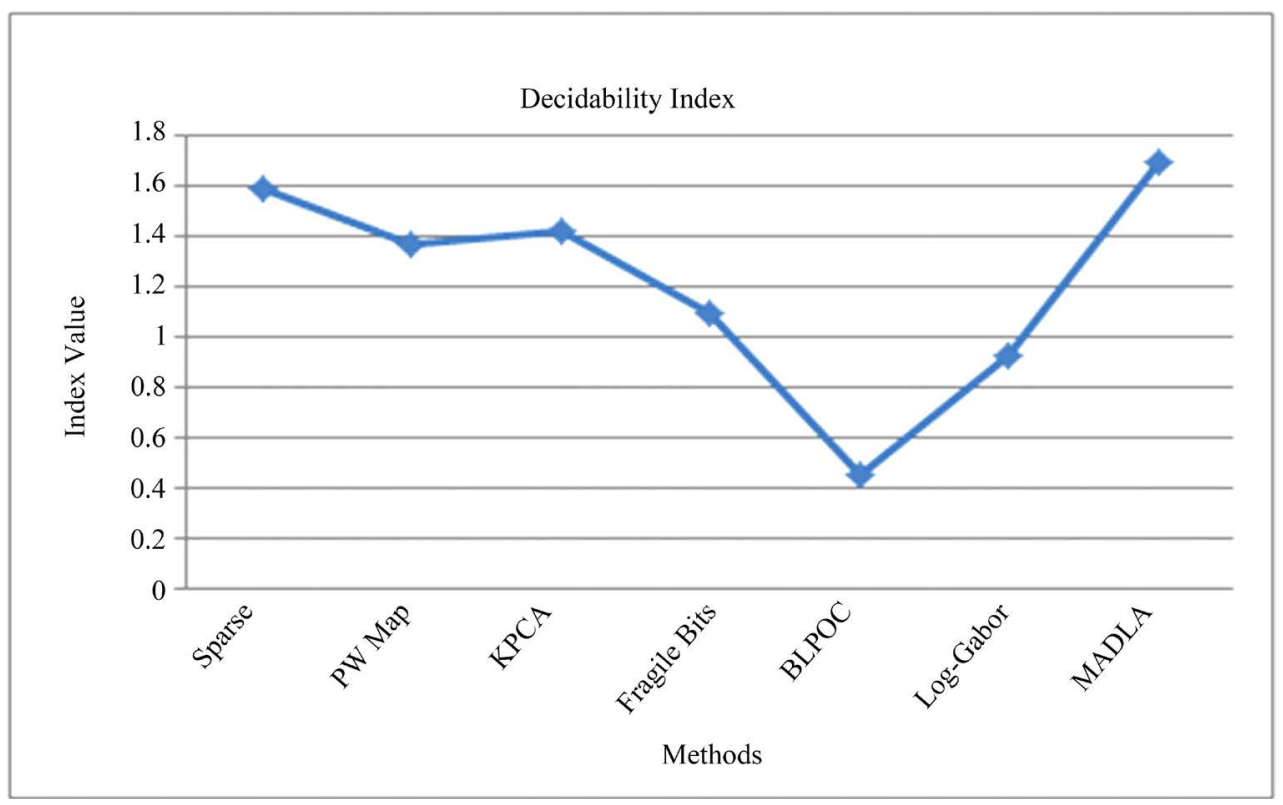

Figure 5. Decidability index comparison. 


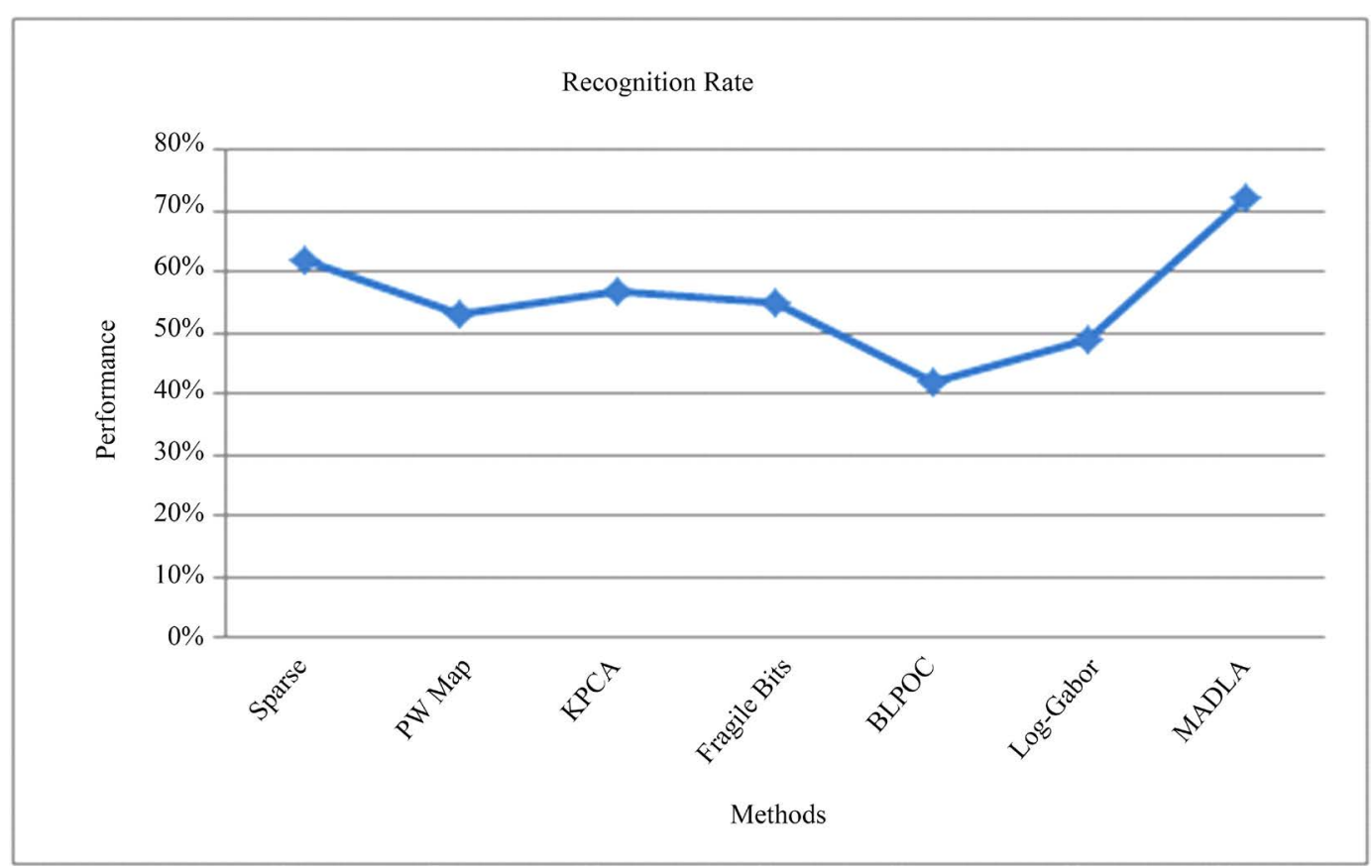

Figure 6. Recognition rate comparison.

Table 1. Comparison of algorithm.

\begin{tabular}{cccc}
\hline Methods & Error Rate & Decidability Index & Recognition Rate \\
\hline Sparse & 0.1922 & 1.5842 & $62 \%$ \\
PWMap & 0.2608 & 1.3700 & $53 \%$ \\
KPCA & 0.2193 & 1.4176 & $57 \%$ \\
Fragile Bits & 0.2534 & 1.0923 & $55 \%$ \\
BLPOC & 0.4022 & 0.4528 & $42 \%$ \\
Log-Gabor & 0.2745 & 0.9266 & $49 \%$ \\
MADLA & 0.1837 & 1.6892 & $72 \%$ \\
\hline
\end{tabular}

will extend the proposed system with encode of iris image and develop a system for ensure security and cost consumption.

\section{References}

[1] Si, Y.L., Mei, J.Y. and Gao, H.J. (2012) Novel Approaches to Improve Robustness, Accuracy and Rapidity of Iris Recognition Systems. IEEE Transactions on Industrial Informatics, 8, 110-117. http://dx.doi.org/10.1109/TII.2011.2166791

[2] Viriri, S. and Tapamo, J.-R. (2015) Improving Iris-Based Personal Identification Using Maximum Rectangular Region Detection. School of Computer Science, University of KwaZulu-Natal, Durban.

[3] Daugman, J. (2002) How Iris Recognition Works. Proceedings of 2002 International Conference on Image Processing, 1, I-33-I-36. http://dx.doi.org/10.1109/icip.2002.1037952

[4] Mattar, E. (2013) Principal Components Analysis Based Iris Recognition and Identification System. International Journal of Soft Computing and Engineering (IJSCE), 3, 430.

[5] da Costa, R.M. and Gonzaga, A. (2012) Dynamic Features for Iris Recognition. IEEE Transactions on Systems, Man, and Cybernetics, Part B: Cybernetics, 42, 1072-1082. http://dx.doi.org/10.1109/TSMCB.2012.2186125

[6] Pillai, J.K., Puertas, M. and Chellappa, R. (2014) Cross-Sensor Iris Recognition through Kernel Learning. IEEE Transactions on Pattern Analysis and Machine Intelligence, 36, 73-85. http://dx.doi.org/10.1109/TPAMI.2013.98

[7] Sarikaya, R., Hinton, G.E. and Deoras, A. (2014) Application of Deep Belief Networks for Natural Language Under- 
standing. IEEE/ACM Transactions on Audio, Speech, and Language Processing, 22, 778-784. http://dx.doi.org/10.1109/TASLP.2014.2303296

[8] Chen, Y.S., Lin, Z.H., Zhao, X., Wang, G. and Gu, Y.F. (2014) Deep Learning-Based Classification of Hyperspectral Data. IEEE Journal of Selected Topics in Applied Earth Observations and Remote Sensing, 7, 2094-2107. http://dx.doi.org/10.1109/JSTARS.2014.2329330

[9] Proença, H. and Alexandre, L.A. (2006) Iris Segmentation Methodology for Non-Cooperative Recognition. IEE Proceedings Vision, Image and Signal Processing, 153.

[10] Liam, L., Chekima, A., Fan, L. and Dargham, J. (2002) Iris Recognition Using Self-Organizing Neural Network. IEEE 2002 Student Conference on Research and Developing Systems, Malaysia, 169-172.

[11] Du, Y., Ives, R., Etter, D., Welch, T. and Chang, C. (2004) A New Approach to Iris Pattern Recognition. SPIE European Symposium on Optics/Photonics in Defense and Security, London, October 2004.

\section{Submit or recommend next manuscript to SCIRP and we will provide best service for you:}

Accepting pre-submission inquiries through Email, Facebook, Linkedin, Twitter, etc A wide selection of journals (inclusive of 9 subjects, more than 200 journals)

Providing a 24-hour high-quality service

User-friendly online submission system

Fair and swift peer-review system

Efficient typesetting and proofreading procedure

Display of the result of downloads and visits, as well as the number of cited articles

Maximum dissemination of your research work

Submit your manuscript at: http://papersubmission.scirp.org/ 